\title{
Study of the effect of ultrafast heating on the structure and shape of the gas phase synthesized $\mathrm{Cu}$ nanoparticless
}

\author{
I.V. Chepkasov ${ }^{1 \dagger}$, Yu.Ya. Gafner ${ }^{1}$, E.D. Kurbanova², V.A. Polukhinn ${ }^{2,3}$ \\ †ilya_chepkasov@mail.ru \\ ${ }^{1}$ Khakas State University, 90 Lenina St., 655017, Abakan, Russia \\ ${ }^{2}$ Institute of Metallurgy, Ural Branch, RAS, 101 Amundsen St., 620016, Yekaterinburg, Russia \\ ${ }^{3}$ Institute of Material Studies and Metallurgy, Ural Federal University, 28 Mira St., 620062, Yekaterinburg, Russia
}

\begin{abstract}
Molecular dynamics method using the tight-binding potential to carry out simulation of ultrafast heating of the synthesized particles from the gas phase to a temperature $T=600 \mathrm{~K}$ and $T=900 \mathrm{~K}$, at which the particles were kept about $10 \mathrm{~ns}$. As a result of the simulation revealed that the method of ultrafast heating the particles to high temperatures virtually eliminates the possibility of a clusters of defective education, but as a result of the heat treatment, the some of investigated particles can disconnect (burst) into smaller clusters.
\end{abstract}

Keywords: computer modeling, copper nanoparticles, gap particles, structure, gas phase.

\section{Introduction}

The recently grown interest in the development of new and improvement of the available methods for fabrication of nanodispersed materials is due to the fact that it is this class of processing substances that finds an ever broadening application as the initial raw material in the production of the most diverse ceramic and composite compounds, superconductors, data storage media, filters, catalysts [1, 2] and nanoelectronic devices [3]. In the first place, this is conditioned by the fact that during a transition from the bulk phase to a state with a particle size of below $100 \mathrm{~nm}$ a number of fundamental properties of a material change, such as thermal and electrical conductivity, thermal capacity, chemical reactivity, and many others.

In the whole range of the metallic nanoparticles in use today, copper clusters hold a special place. These particles possess many unique properties and are comparatively inexpensive to manufacture. In the context of their electrically conductive properties, nanometer-sized copper particles can compete even with silver and can be applied as contacts in high-temperature electronics [4].

Currently, there are many methods to produce metal nanoparticles, such as mechanochemical grinding methods, plasma-chemical methods, some variations of chemical, photochemical and radiation reduction, the method of condensation from the gas phase [5]. Among a great variety of methods for fabrication of metallic nanoparticles, one of the promising synthesis methods is evaporation-condensation of metals in an inert gas atmosphere. A simple scalability to industrial standards and a high cleanliness of a material with the required properties (electrical conductivity, strength, ductility) favorably distinguish particles synthesized by the gas-phase method from particles produced by other methods, such as mechanical milling.
However, in most methods for fabrication of nanoparticles such conditions may be created when particles of a nonspherical shape form as a result of synthesis. Such randomshaped formations may consist of tens of united primary entities, formed at the early stages of synthesis. The nonspherical cluster formations, produced in large quantities, are not rejects in the production of perfect spherical particles, and they can also be used in various areas of nanotechnology as catalysts and battery electrodes [6-8].

However, if in the process of synthesis it is necessary to produce perfect spherical particles, actively applied, for instance, in data storage media [9], heat treatment is used at the final stage of production. The produced agglomerates and aggregates are subjected to thermal sintering, leading to compaction of nanoformations to spherical particles. But to create the most effective process of thermal action, precise information is required about the morphological changes in the investigated particles throughout the whole process of heating.

To obtain detailed information about the processes of synthesis and subsequent thermal sintering of nanopowders, computer simulation techniques are increasingly used. In the present work, to identify the main features of heat treatment of particles, condensed from the high-temperature gas phase, molecular dynamics simulation using the tight-binding potential [10] was performed, of the heating processes of copper clusters in the framework of the Nose-Hoover thermostat. In our opinion, this thermostat suits best for simulation of the processes of heating and cooling of isolated particles.

\section{Results and discussion}

Copper nanoparticles, taken for identification of the most effective post-condensation methods of heat treatment, were synthesized from the gas phase at the first stage of 
simulation. The condensation process was implemented with three different cooling rates $U=0.005 \mathrm{ps}^{-1}$, $U=0.025 \mathrm{ps}^{-1}$ and $U=0.05 \mathrm{ps}^{-1}$, and two final temperatures $T_{f}=373 \mathrm{~K}$ and $T_{f}=77 \mathrm{~K}$. More detailed information about the method of simulation of the gas-phase synthesis process and the obtained simulation results is given in $[11,12]$.

Two main techniques of heat treatment of synthesized nanopowders were studied, namely step-by-step heating and ultrafast heating. The results of the effect of step-bystep heating were presented earlier in [13]. Let us address only the main conclusions made from the results of the step-by-step heating study. As a result of a stepwise increase in the particles temperature from 100 to $1200 \mathrm{~K}$ (with a step of $100 \mathrm{~K}$ ), perfect ordering of the internal structure and external shape in the process of heat treatment was observed in only $70 \%$ of the cluster array under study. The remaining $30 \%$ of the clusters in the process of thermallyactivated relaxation could not form a unified shape and structure, and constituted competing parts of one particle with comparable surface energies and chemical potential energies.

In order to exclude such a behavior of conglomerates during thermal action, for this work the method of ultrafast heating of particles to relatively high temperatures of $T=600 \mathrm{~K}$ and $T=900 \mathrm{~K}$ was selected. In our opinion, this method, owing to high temperatures and, correspondingly, short sintering times $(t=10 \mathrm{~ns})$, can lead to a rather quick formation of a particle's unified external shape and ordered internal structure, without formation of significant defects within the cluster.

All the particles produced during simulation of copper atoms condensation from the gas phase were subjected to the procedure of ultrafast heating and thermal relaxation. During the simulation of six condensation variants (with various final temperatures and cooling rates), in total 136 copper clusters of
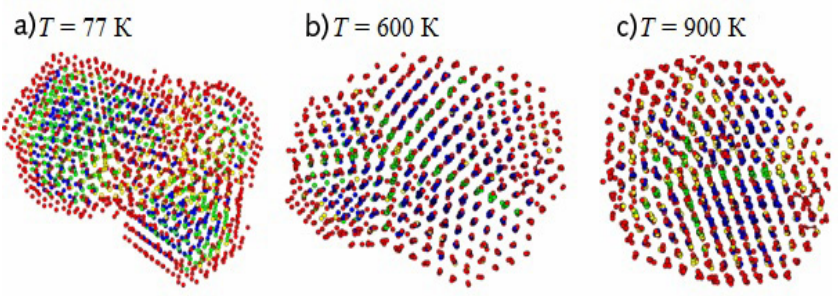

Fig. 1. Images of the cluster $\mathrm{Cu}_{1771}$ in the initial state (a) and after heat treatment at $T=600 \mathrm{~K}(\mathrm{~b})$ and $T=900 \mathrm{~K}(\mathrm{c})$.

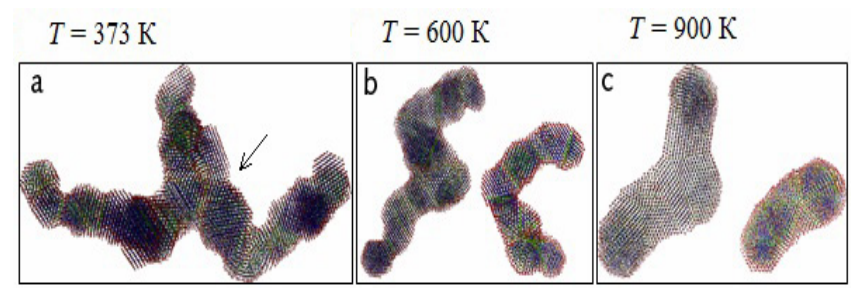

Fig. 2. Images of the cluster $\mathrm{Cu}_{17916}$ in the initial state (a) and after heat treatment at $T=600 \mathrm{~K}(\mathrm{~b})$ and $T=900 \mathrm{~K}(\mathrm{c})$. various shapes and structures with a size of 81 to 37140 atoms were synthesized. As a result of the conducted simulation, it was proved that the method of ultrafast heating of particles to the heat treatment temperatures does practically exclude the possibility of emergence of defect formations in clusters subjected to heating. For instance, the cluster $\mathrm{Cu}_{1771}$, already investigated earlier and displayed in Fig.4 in the paper [13], which had not formed a united particle in the course of stepby-step heating, was subjected to ultrafast heating to high temperatures ( $T=600 \mathrm{~K}$ and $T=900 \mathrm{~K}$ ).

From the images presented in Fig. 1 displaying $\mathrm{Cu}_{1771}$, it is clearly visible that after the conducted heat treatment this cluster assumed a practically perfect spherical shape and completely reconstructed its structure. As a result, from a disordered conglomerate that had several local competing structures leading to emergence of defect formations during step-by-step heat treatment, the cluster $\mathrm{Cu}_{1771}$ turned into a perfect particle, suitable for application in high-precision instruments, in which the main criterion of efficiency is the degree of order of the internal structure and the degree of perfection of the external shape.

However, the process of ultrafast heating of particles to the selected heat treatment temperatures does not always result in formation of a united, perfectly ordered particle. As a result of heat treatment, some of the particles broke down (burst) into smaller clusters not connected with each other. The number of clusters, which were subjected to heat treatment and then «burst», varied from $5 \%$ to $83 \%$, depending on the parameters of synthesis of these particles from the gas phase.

Figure 2 shows an example of a particle which «burst» during thermal action. Such a behavior of particles synthesized from a gas atmosphere and subjected to heat treatment at rather high temperatures is observed also in experimental works on sintering of silver clusters. For

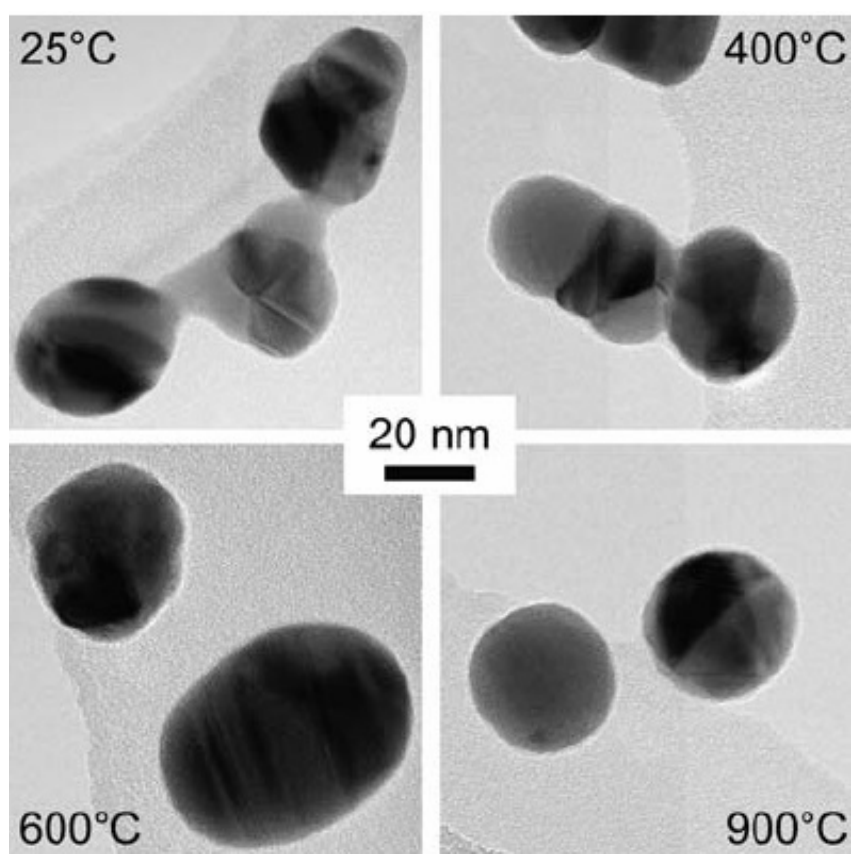

Fig. 3. Electron-microscopy images showing sintering of silver nanoparticles at various temperatures [14]. 
instance, in the work [14] the authors investigated the variations in the external shape of primary silver particles as a result of sintering at various temperatures. In the course of the conducted procedure, the primary particle divided into separate clusters, which subsequently formed several perfect spherical particles not connected with each other (Fig.3).

In order to account for such a behavior of the particles during heat treatment, a detailed analysis was performed, of the most typical representatives of the united and «burst» particles. As the most typical examples, this work mentions four particles from a group with a particle size from 6000 to 10000 atoms and two particles from a group with a particle size of 15000 to 18000 atoms. All the particles taken for comparative analysis were produced in systems with a final condensation temperature of $T_{f}=373 \mathrm{~K}$. In the selected examples, the absence of clusters synthesized at a final temperature of $T_{f}=77 \mathrm{~K}$ is conditioned by the fact that at such a condition of gas-phase synthesis the percentage of the

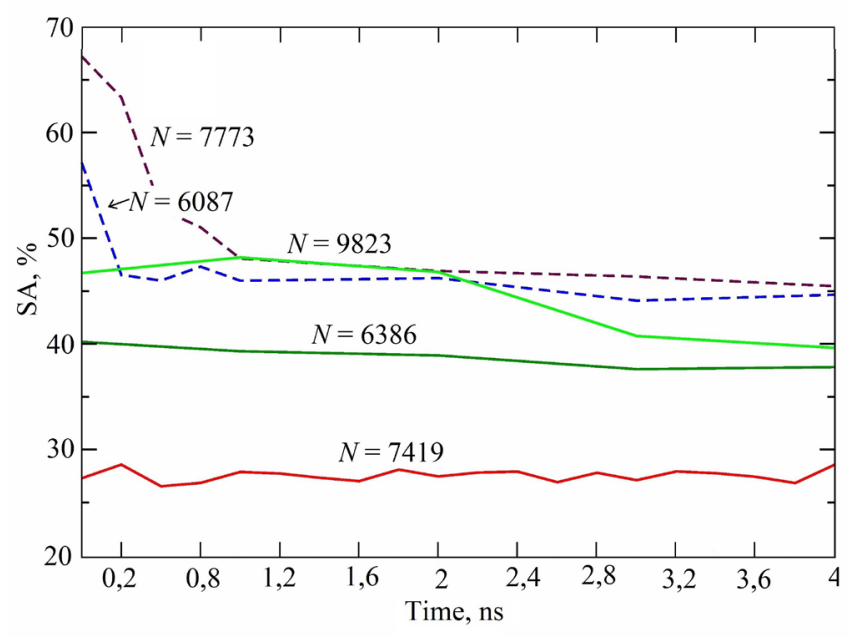

a

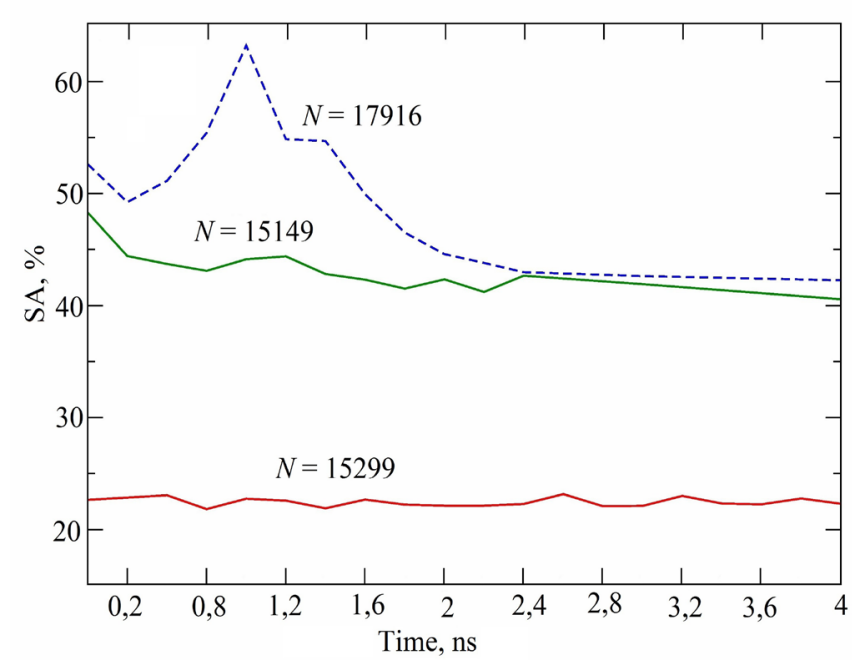

b

Fig. 4. Time dependencies of the percentage of surface and "defect" atoms (SA) for copper clusters from the group of medium particles (6000 to 10000 atoms) (a) and clusters from the group of large particles (15000 to 18000 atoms) (b). The dependencies of the burst clusters are denoted by a dashed line. $\mathrm{Cu}_{7419}$ and $\mathrm{Cu}_{15299}$ are perfect spherical copper clusters cut out from an $f c c$-lattice. «burst» clusters was only 5-9\%, which could be caused by some stochastic processes.

With a view to define the various scenarios of cluster rearrangement, in the considered particles the time dependencies were studied, of the surface atoms fraction and of the fraction of atoms referring to local defect structures (the coordination number below 12). The values of these quantities were summed and represented by a single quantity SA whose time dependencies for the first four ns of heating at $T=600 \mathrm{~K}$ are given in Fig. 4. As can be seen from the graphs, at the initial stage of heating for the «burst» and united particles with a medium size (6000-10000 atoms) the SA values differ by $17-21 \%$. In about $0.6 \mathrm{~ns}$ after the start of heating, the SA values for the «burst» particles decline rather sharply and settle in the vicinity of the SA values for the united clusters. Such a behavior is related to the fact that by that time the primary particles under investigation, poorly connected with each other, had already disintegrated into secondary clusters which, in their turn, optimized the structure rather quickly.

Such a divergence in the SA values at the initial stage of heat treatment is significant and may lead, due to intracluster restructuring, to a complete dissociation of particles, since in the process of thermal action each cluster, guided by the law of minimum energy, tries to optimize its structure, which, in the case of particles having a large quantity of surface atoms and atoms with a local disordered structure, leads to competitive struggle between growth centers of local atomic organizations.

A slightly different situation is observed in the case of the clusters $\mathrm{Cu}_{17916}$ and $\mathrm{Cu}_{15149}$. Small differences in the SA values at the initial stage of heating $(4.5 \%)$ for these particles, apparently, were not supposed to lead to large differences in cluster restructuring (Fig.4b). However, in the process of thermal action in the particle $\mathrm{Cu}_{17916}$ occurs structure destruction of a rather large layer connecting two main cluster particles, which results in a sharp rise in the SA values during the first ns of heating. The restructuring of this disordered area subsequently, at $2.2 \mathrm{~ns}$, leads to the dissociation of the particle into secondary clusters with a size of 5760 and 12156 atoms.

Also, as a result of the conducted instant heating to the heat treatment temperatures, 136 copper nanoparticles synthesized from the gas phase were investigated in detail in the context of the shape of these particles at the final stage of heating at $T=900 \mathrm{~K}$. Detailed information on the initial and final shape of the clusters under study is presented in table 1 . It can be clearly seen that in all the condensation systems, heat treatment considerably improved the shape of the synthesized particles, and the percentage of spherical particles increased by $17 \%-48 \%$ depending on the parameters of particle synthesis. Although in the system with a final condensation temperature of $T_{f}=373 \mathrm{~K}$ and a cooling rate of $U=0.005 \mathrm{ps}^{-1}$ the number of spherical particles after heat treatment is equal to zero, this kind of a result may not be regarded as a complete disappearance of spherical clusters in the system, since a large percentage of the particles were subjected to «bursting», and spherical clusters were present in the system, but in the form of secondary particles (products of «bursting») of the primary clusters.

It also follows from analysis of table 1 that the large 
Structure and shape of $\mathrm{Cu}$ nanoclusters synthesized from the gas phase, before and after heat treatment

\begin{tabular}{|c|c|c|c|c|c|c|c|}
\hline \multirow{2}{*}{$\begin{array}{c}\text { Cooling rate } \\
\left(U, \mathrm{ps}^{-1}\right)\end{array}$} & \multirow{2}{*}{$\begin{array}{c}\text { Temperature } \\
\left(T_{f}, K\right)\end{array}$} & \multirow{2}{*}{$\begin{array}{c}\text { Number } \\
\text { of } \\
\text { clusters }\end{array}$} & \multicolumn{2}{|c|}{ Initial shape $\left(\right.$ at $\left.T_{f}, K\right)$} & \multicolumn{3}{|c|}{$\begin{array}{c}\text { Final shape } \\
\text { (at } \mathrm{T}=900 \mathrm{~K})\end{array}$} \\
\cline { 5 - 9 } & & Sphere & Agglomerate & Sphere & \multicolumn{2}{|c|}{ Agglomerate } & Bursting \\
\hline 0,05 & 77 & 46 & $23,92 \%$ & $76,08 \%$ & $63,04 \%$ & $28,26 \%$ & $8,70 \%$ \\
\hline 0,025 & 77 & 35 & $25,72 \%$ & $74,28 \%$ & $74,28 \%$ & $20,00 \%$ & $5,72 \%$ \\
\hline 0,005 & 77 & 17 & $41,17 \%$ & $58,83 \%$ & $58,82 \%$ & $35,30 \%$ & $5,88 \%$ \\
\hline 0,05 & 373 & 19 & $10,53 \%$ & $89,47 \%$ & $42,10 \%$ & $21,05 \%$ & $36,85 \%$ \\
\hline 0,025 & 373 & 13 & $15,39 \%$ & $84,61 \%$ & $46,15 \%$ & $15,39 \%$ & $38,46 \%$ \\
\hline 0,005 & 373 & 6 & $16,66 \%$ & $83,34 \%$ & $0 \%$ & $16,66 \%$ & $83,84 \%$ \\
\hline
\end{tabular}

percentage of agglomerates (worm-like and dumbbell-like clusters), present in the systems at the initial condensation stage, diminishes considerably after heat treatment. As a result of the thermally-activated cluster restructuring, these agglomerates pass into the fraction of spherical and «burst» particles. In the systems with a final temperature of $T_{f}=77 \mathrm{~K}$ a tendency is observed for a widespread integration of agglomerates into spherical particles, with formation of a small percentage of «burst» clusters. On the other hand, the opposite tendency is observed in the systems with a final temperature of $T_{f}=373 \mathrm{~K}$, i.e. a widespread «bursting» of agglomerates during heat treatment, with formation of a small percentage of united spherical particles, or even their absence.

\section{Conclusions}

From the results of the performed simulation, it can be concluded that the exceeding of the $50 \%$ threshold quantity of surface atoms and atoms having a local disordered structure may lead to the burst of a particle into secondary particles in the process of instant heating, due to rapid cluster restructuring. Thus, the use of these clusters in hightemperature chemical reactions may be severely hampered due to a rapid dissociation of the used particles, and, consequently, a sharp decline in the catalytic efficiency of the used nanomaterial.

This study was performed within the framework of the State Task of the Ministry of Education and Science of the Russian Federation and supported in part by the Russian Foundation for Basic Research (project no. 13-02-98000-r_sibir'_a)

\section{References}

1. A.P. Weber, P. Davoodi, M. Seipenbusch, G. Kasper. Journal of Nanoparticle Research. 5, 293 (2003).

2. A.P. Weber, M. Seipenbusch, C. Thanner, G. Kasper. Journal of Nanoparticle Research. 1, 253 (1999).

3. H. Fissan, M. K. Kennedy, T. J. Krinke, F.E. Kruis. Journal of Nanoparticle Research. 5, 299 (2003).

4. Yiying Yao, Zheng Chen, Guo-Quan Lu, Khai D.T. Ngo. IEEE Transactions on Components. Packaging and Manufacturing Technology. 2, 539 (2012).

5. K. Binder. Monte Carlo methods in statistical physics: transl. from English. Edited by V.N. Novikov. M. Mir. (1982) 399 p. (in Russian)

6. S.E. Pratsinis. AIChE Journal. 56, 3028 (2010).

7. H. Ogawa, M. Nishikawa, A. Abe. Journal of Applied Physics. 53, (1982) p. 4448-4455.

8. S.Y. Chew, T.J. Patey, O. Waser, S.H. Ng, R. Buechel, A. Tricoli, F. Krumeich, J. Wang, H. K. Liu, S. E. Pratsinis, P. Novak. Journal of Power Sources. 189, 449 (2009).

9. Yu.I. Golovin. Introduction to nano-engineering. M. Mashinostroenie. (2007) 496 p. (in Russian)

10. F. Cleri, V. Rosato. Phys. Rev. B48, 22 (1993).

11. I. V. Chepkasov, Yu. Ya. Gafner, S.L. Gafner. Letters on Materials. 1, 107 (2011). (in Russian)

12. I. V. Chepkasov, Yu. Ya. Gafner. Fundamental'nye problemy sovremennogo materialovedenia (Basic Problems of Material Science). 9, 353 (2012). (in Russian)

13. I.V. Chepkasov, Yu.Ya. Gafner, A.R. Khalikov, Letters on Materials. 4(3), 205 (2014). (in Russian)

14. M.N. A. Karlsson, K. Deppert, L.S. Karlsson, M.H. Magnusson, J.O. Malm, N.S. Srinivasan. Journal of Nanoparticle Research. 7, 43 (2005). 